\title{
Neurocysticercosis Association with Cognitive and Aberrant Behavioural Symptoms: A Case Report and Review
}

\author{
Rohit Verma ${ }^{1}$, Kuljeet Singh Anand ${ }^{2}$, Mina Chandra ${ }^{1}$, Neha Prakash ${ }^{2}$, Ankur Sachdeva ${ }^{1}$ \\ ${ }^{1}$ Department of Psychiatry, Post Graduate Institute of Medical Education and Research, \\ Dr Ram Manohar Lohia Hospital, New Delhi, India \\ ${ }^{2}$ Department of Neurology, Post Graduate Institute of Medical Education and Research, \\ Dr Ram Manohar Lohia Hospital, New Delhi, India \\ E-mail: rohit.aiims@gmail.com
}

Received June 10, 2011; revised July 7, 2011; accepted August 26, 2011

\begin{abstract}
Neurocysticercosis (NCC) is a leading cause of seizures and epilepsy worldwide. Its clinical manifestations are varied, non specific and pleomorphic, depending on multiple factors. Since NCC mimic large number of neurological disorders, it is important that clinicians should be familiar with these rare presentations to avoid delay in diagnosis and management. We herein describe an unusual case of multiple NCC's involving entire cortex with only cognitive and behavioural manifestations and review pertinent literature.
\end{abstract}

Keywords: Neurocysticercosis, Dementia, Neuropsychiatric

\section{Introduction}

Neurocysticercosis (NCC) is the common parasitic infestation of the central nervous system worldwide. The clinical manifestations of NCC are varied and depend on the topography, number, the size and stage of lesions, as well as the status of the host's immune response to the parasite [1]. While some cases of NCC may remain asymptomatic, most have been reported to present seizures, headache, raised intra cranial tension (ICT) due to blockage of CSF flow, stroke, neuropsychiatric symptoms, ophthalmologic and endocrinological manifestations [2]. Dementia, being the first and only presenting symptom, has been an unusual association with NCC. We herein describe an unusual case of multiple NCC's involving entire cortex with only cognitive and behavioural manifestations and review pertinent literature.

\section{Case Report}

A 44 year old non-diabetic, normotensive non-vegetarian male of middle socio economic status, educated up to higher secondary, presented acute onset behavioral disturbances and forgetfulness for two and half month. The symptoms started after a mild continuous fever of 4 days followed by $\mathrm{B} / \mathrm{L}$ fronto-temporal headache which was followed few days later by abrupt onset inappropriate behaviour like walking in circles on bed, patting his body parts, and shouting inappropriately. Patient would not stop despite the efforts of family members. He received some medications from a nearby hospital (records not available) but they showed no improvement but only put patient to sleep. In a few days, patient started giving inappropriate response to asked questions, irrelevant and abusive talking. Patient had become irritable and started exhibiting symptoms of forgetfulness like difficulty finding rooms in his own house, unable to find way back home from known places. Patient seemed confused most of the time and disoriented to time and place. His activities of daily living were affected and he needed constant nagging to carry out work. He was also not able to maintain hygiene. Patient would wander inside and outside the house for no reason. His sleep duration was decreased with difficulty in initiating sleep.

There was no history suggestive of coronary artery disease, cerebro-vascular disease, trauma, blood transfusion, joint pain, rashes, photosensitivity, seizure-like activity, and substance use. The patient did not give any history of loss of consciousness, vomiting, dysphagia, dysarthria, gait ataxia or any weakness. There was no history of any perceptual disturbances, delusion, suspiciousness, or depressed mood. There was no past or family history of any similar, psychiatric or neurological illness. His premorbid personality was well adjusted.

The general physical examination was normal. Visual acuity, including pupils, intraocular pressure and ocular fundi were normal. Both upper and lower limb bulk, power, 
reflex and plantar response were normal. Sensory and cerebellar examinations were also normal. Cranial nerveswere intact. Extrapyramidal system showed no abnormality. No involvement of other system was found on examination. On mental state examination, rapport was formed easily. There was no abnormality in psychomotor activity and speech was relevant, coherent, with normal tone and productivity. Affect was euthymic with no perceptual or abnormalities in thought-form, flow, content, or possession. Attention was arousable but ill-sustained. He was oriented to person but not to time or place. Immediate, recent and remote memory was impaired with confabulations. Judgement was impaired with no insight into the illness. His mini-mental state examination (MMSE) score was 10 [3]. On lobar function testing using Strub and Black scheme, impairment was observed in working and episodic verbal memory, executive functions, verbal fluency, constructive praxis, and visual-spatial orientation. Clinical Dementia Rating scale score was 2 suggesting moderate dementia [4]. The Hamilton Depression Rating Scale score was 7 , thus limiting the influence of negative affect over cognitive integrity [5].

Complete blood count, blood sugar, electrolyte, LFT, KFT, and lipid profile were normal. X-ray chest, EEG and echocardiogram were normal. On magnetic resonance imaging (MRI) of brain, bilateral cerebral hemispheres, cerebellum and brain stem region a multitude of tiny cystic lesions of a similar nature were seen (Figure 1). There was adjoining hyperintensity on T2 and FLAIR images in few of the lesions. There was involvement of bilateral gangliocapsular region, thalami, and caudate nucleus. Bilateral parotid showed multiple tiny similar cystic lesions. Bilateral mastoids showed hyperintensity within. Ventricles were normal with basal cisterns, sylvian fissures and intracranial vascular flow voids preserved. Ophthalmic evaluation was normal including B-scan. Enzyme linked immunoabsorbent assay (ELISA) of the cerebrospinal fluid (CSF) confirmed NCC infection.

\section{Discussion}

Common infective causes of dementia include prion

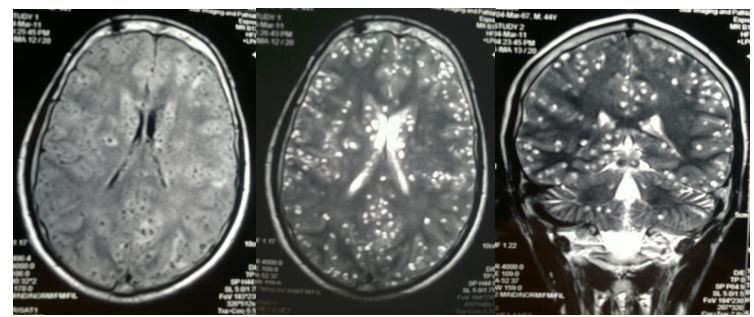

Figure 1. (MRI brain scan showing bilateral cerebral hemispheres, cerebellum and brain stem region were seen studded with multiple 1 - 2 mm size ring lesion with cystic centre and eccentric dark signal foci within). disease, herpes virus, toxoplasmosis, cryptococcus, cytomegalovirus, neurocysticercosis, syphillis, and borelia [6]. NCC is an infestation of the brain by Taenia solium, the tissue-invading larval form of the pork tapeworm. The larvae are introduced into the body by the ingestion of Taenia solium eggs in fecally contaminated food or water. Once ingested, the eggs hatch in the duodenum and release larvae. The larvae then penetrate intestinal mucosa and enter the circulatory system, where they eventually localize as cysts within the skeletal muscles, the eyes, or the brain [2,7]. NCC is mainly located in gray matter or at the junction of gray and white matter with a rich blood supply. In this intra-parenchymal NCC, seizure is the most common clinical manifestation. Extraparenchymal NCC refers primarily to infection of the ventricles and subarachnoid spaces [2]. Clinical presentation is of more aggressive behaviour as compared to the parenchymal lesions, and generally, the prognosis of these patients is worse [8]. Leptomeningeal involvement of NCC may cause intrasellar arachnoiditis, which is one of the most severe complications of NCC, associated with hydrocephalus, multiple cranial nerves dysfunction, and death [9]. Subarachnoid involvement of NCC is in $3.5 \%$ of the patients with NCC present with parasites in vesicles of the subarachnoid space, presented with visual and hormonal impairment by direct compression of the hypophyseal stem and the optic nerves [8]. Tuberculous granuloma, microabscess, focal meningoencephalitis, neoplasms, and vascular lesions should be considered in the differential diagnosis [10].

Prevalence of NCC approaches $4 \%$ in rural areas of the developing world [11]. Although neurocysticercosis can cause almost any neurological symptom, late-onset epilepsy and intracranial hypertension are its most common clinical manifestations [2]. Cognitive and behavioural deteriorations in NCC are more commonly associated with hydrocephalus or multiple lesions $[2,8]$.

Psychiatric symptoms have been frequently associated with NCC; severity of psychiatric symptoms may correlate with treatment of NCC with anti-parasitic drugs, associated with an increase in CNS inflammation [11]. The mental changes most frequently reported are: confusion, disorientation, memory loss, hallucinations, psychomotor incoordination, progressive deterioration of language ability, and mental deterioration [12].

Forlenza et al. [11] reviewed 38 cases of neurocysticercosis and reported psychiatric illness and cognitive decline in $65.8 \%$ and $87.5 \%$ of cases, respectively. Amongst psychiatric illness, depressive disorders and psychosis was seen in $52.6 \%$ and $14.2 \%$ of cases, respectively. Progression of disease and intracranial hypertension correlated with higher levels of psychiatric comorbidity. Previous episodes of depression correlated with recurrence of depression in active disease. Sotelo et al. [13] reviewed 753 cases 
and reported intellectual deterioration in $15.8 \%$, psychosis in $4.7 \%$, and behavioral disturbance in $2.7 \%$. These symptoms were more common with hydrocephalus or multiple lesions. In a review of 23 cases, Loo and Braude found that in $56 \%$, the presenting symptom was psychiatric, while abnormal mental status examination results were a presenting sign in 43\% [14]. McCormick et al. [15] reviewed 127 cases and found that 38\% had intracranial hypertension; $10 \%$ had increased intracranial pressure and dementia. In a review of 17 cases, Grisolia and Wiederholt reported that $18 \%$ exhibited altered mental status because of increased intracranial pressure [16]. Scharf reviewed 238 cases; at some point during the course of illness, 33\% experienced altered mental status [17]. Two percent presented with dementia, 3\% with psychosis. Wadia et al. reported three cases of disseminated cysticercosis (involving brain and muscle tissue); all exhibited dementia, and two cases experienced psychosis [18]. There are also case reports of varied presentation of NCC including pure motor hemiparesis, alexia and manic episodes [19-22].

Dixon and Lipscomb reported schizophrenic and maniclike episodes as possible initial signs of neurocysticercosis and found only 5 cases of true dementia in 450 patients (1.1\%) [23]. Andrade et al. [24] in a cross-sectional controlled study reported dementia in 5 (12.5\%) of 40 patients with NCC and attributed this to a synergistic interaction of cysticercus lesions and local inflammation. In their view, antiepileptic drug use and seizure frequency could not account for the cognitive features. They found no correlation between cognitive scores and number, localization, or type of NCC lesions on MRI. Rosselli et al. [25] reported a severe case of dementia associated with a parenchymous miliary neurocysticercosis without motor seizures whose EEG showed epileptic-like focal discharges. The clinical presentation was of headaches, associated with apathy, poor school work and finally hallucinations, confusion and excitement in apparently paroxysmal episodes. Diagana et al. [26] studied cognitive impairment in an Ecuadorian urban Andean community known to be an endemic zone for Taenia solium taeniasis and cysticercosis. In a sample of 227 adults over the age of 40 years assessed using Mini mental state tests (MMS), the overall prevalence of cognitive impairment was 8.4\% (19/227). In the elderly above the age of 75 years the prevalence of cognitive impairment was $23.5 \%$. The authors linked this high prevalence of dementia to the endemic nature of Taenia solium taeniasis and cysticercosis in this community. The authors postulated that parasitic encephalopathy which was present in $20 \%$ persons in this Andean community could play a part in early damage of cognitive function and hence result in such high prevalence of dementia. The single case report of NCC presenting only dementia as per our knowledge was by
Jha and Ansari [27].

Various pathophysiological mechanisms have been suggested to explain the symptom production by NCC infection. Fuentes suggested that psychiatric symptoms associated with NCC were not due to the direct effect of the parasite on a definite region of the brain but were rather related to mechanical alterations in CSF pressure. Moreover, inflammatory injury of the brain parenchyma may also play some role [28]. Parasites may induce expression of several classes of genes in the CNS of the host and exceeds the gene expression seen in inflammatory processes. These genes encode proteins or neuropeptides involved in the regulation of vital physiologic and behavioral processes, which have led to the behavioral manipulation hypothesis $[29,30]$. It has been observed that NCC affects brain regions that are distant from the location of the cysticerci [31]. Thus location of lesion itself appears insufficient to cause cognitive changes.

Dementia syndrome observed in patients with NCC could be a combined effect, resulting from multiple parasitic and vascular lesions, disrupting frontal-parietal-temporal networks related to intellectual functioning in patients with vulnerable brains (because of repeated epileptic seizures, low educational levels, advanced age) [32]. One study suggests that dementia occurs frequently in patients with untreated NCC, and it is reversible in most cases [32]. Most studies for evaluating cognition in NCC have taken patients having associated seizures and their influence over assessment is indeed a bias.

Dementia as an initial presentation of NCC is a rare occurrence, and even rarer is its occurrence solely. The case described here is of a miliar cysticercosis, affecting both cortical and subcortical structures within the cerebral parenchyma. Our case did not present with any of the common symptoms of NCC and the diagnosis was made on the basis of MRI and ELISA reports.

\section{Conclusions}

We describe a 44-year-old man with a multiple NCC studded over whole cortex. Patients with unusual clinical manifestations should be investigated more thoroughly. NCC should be considered in the differential diagnosis of patients presenting with cognitive and disorganised behavioural symptoms in absence of any other psychopathology even in absence of gross neurological signs. Prompt intervention is also recommended for accurate diagnosis, to prevent progression of the disease, and to avoid ireversible loss of the cognitive functions.

\section{References}

[1] O. H. Del Brutto, V. Rajshekhar, A. C. White Jr., V. C. Tsang, T. E. Nash, O. M. Takayanagui, et al., "Proposed 
Diagnostic Criteria for Neurocysticercosis,” Neurology, Vol. 57, No. 2, 2001, pp. 177-183.

[2] H. H. Garcia and O. H. Del Brutto, "Neurocysticercosis: Updated Concepts about an Old Disease,” Lancet Neurology, Vol. 4, No. 10, 2005, pp. 653-661. doi:10.1016/S1474-4422(05)70194-0

[3] M. F. Folstein, S. E. Folstein and P. R. McHugh, “"Mini-Mental State”: A Practical Method for Grading the Cognitive State of Patients for the Clinician,” Journal of Psychiatric Research, Vol. 12, No. 3, 1975, pp. 189198. doi:10.1016/0022-3956(75)90026-6

[4] J. C. Morris, "The Clinical Dementia Rating (CDR): Current Version and Scoring Rules,” Neurology, Vol. 43, 1993, pp. 2412-2414.

[5] M. Hamilton, “A Rating Scale for Depression,” Journal of Neurology, Neurosurgery and Psychiatry, Vol. 23, No. 1, 1960, pp. 56-62. doi:10.1136/jnnp.23.1.56

[6] O. P. Almeida and N. T. Lautenschlager, "Dementia Associated with Infectious Diseases,” International Psychogeriatric, Vol. 17, Suppl. 1, 2005, pp. 65-77. doi:10.1017/S104161020500195X

[7] A. Carpio, "Neurocysticercosis: An Update," The Lancet Infectious Diseases, Vol. 2, No. 12, 2002, pp. 751-762. doi:10.1016/S1473-3099(02)00454-1

[8] S. Sinha and B. S. Sharma, "Neurocysticercosis: A Review of Current Status and Management," Journal of Clinical Neuroscience, Vol. 16, No. 7, 2009, pp. 867-876. doi:10.1016/j.jocn.2008.10.030

[9] N. Arriada-Mendicoa, M. A. Celis-López, J. HigueraCalleja and T. Corona-Vázquez, "Imaging Features of Sellar Cysticercosis," American Journal of Neuroradiology, Vol. 24, No. 7, 2003, pp. 1386-1389.

[10] V. Rajshekhar, R. P. Haran, G. S. Prakash and M. J. Chandy, "Differentiating Solitary Small Cysticercus Granulomas and Tuberculomas in Patients with Epilepsy. Clinical and Computerized Tomographic Criteria,” Journal of Neurosurgery, Vol. 78, No. 3, 1993, pp. 402-407. doi:10.3171/jns.1993.78.3.0402

[11] O. V. Forlenza, A. H. Filho, J. P. Nobrega, L. dos Ramos Machado, N. G. de Barros, C. H. de Camargo, et al., "Psychiatric Manifestations of Neurocysticercosis: A Study of 38 Patients from a Neurology Clinic in Brazil," Journal of Neurology, Neurosurgery \& Psychiatry, Vol. 62, No. 6, 1997, pp. 612-616. doi:10.1136/jnnp.62.6.612

[12] F. Cendes and M. Ferreira, "Manifestacoes Psiquiatricas da Neurocisticercose,” Jornal Brasileiro de Psiquiatria, Vol. 35, No. 2, 1986, pp. 101-106.

[13] J. Sotelo, V. Geurrero and F. Rubio, "Neurocysticercosis: A New Classification Based on Active and Inactive Forms," Archives of Internal Medicine, Vol. 145, No. 3, 1985, pp. 442-445. doi:10.1001/archinte.145.3.442

[14] L. Loo and A. Braude, "Cerebral Cysticercosis in San Diego: A Review of 23 Cases and a Review of the LiteraTure,” Medicine, Vol. 61, No. 6, 1982, pp. 341-359.

[15] G. F. McCormick, C. S. Zee and J. Heiden, "Cysticercosis Cerebri: Review of 127 Cases,” Archives of Neurology,
Vol. 39, No. 9, 1982, pp. 534-539.

[16] J. S. Grisolia and W. C. Wiederholt, "CNS Cysticercosis,” Archives of Neurology, Vol. 39, No. 9, 1982, pp. 540-544.

[17] D. Scharf, "Neurocysticercosis: Two Hundred ThirtyEight Cases from a California Hospital," Archives of Neurology, Vol. 45, No. 7, 1988, pp. 777-780.

[18] N. Wadia, S. Desai and M. Bhatt, "Disseminated Cysticercosis: New Observations, Including CT Scan Findings and Experience with Treatment by Praziquantel," Brain, Vol. 111, No. 3, 1988, pp. 597-614. doi:10.1093/brain/111.3.597

[19] F. Barinagarrementeria and O. H. Del Brutto, "Neurocysticercosis and Pure Motor Hemiparesis,” Stroke, Vol. 19, No. 9, 1988, pp. 1156-1158. doi:10.1161/01.STR.19.9.1156

[20] B. N. Mishra and S. P. Swain, "Psychiatric Morbidity Following Neurocysticercosis," Indian Journal of Psychiatry, Vol. 46, No. 3, 2004, pp. 268-269.

[21] A. Verma, N. N. Singh and S. Misra, "Transitory Alexia without Agraphia: A Disconnection Syndrome Due to Neurocysticercosis," Neurology India, Vol. 52, No. 3, 2004, pp. 378-379.

[22] D. K. Pal, A. Carpio and J. W. A. S. Sander, "Neurocysticercosis and Epilepsy in Developing Countries," Journal of Neurology, Neurosurgery \& Psychiatry, Vol. 68, No. 2, 2000, pp. 137-143. doi:10.1136/jnnp.68.2.137

[23] H. B. F. Dixon and F. M. Lipscornbe, "Cysticercosis: An Analysis and Follow-Up of 450 Cases,” Medical Research Council Special Report Series No. 299, Her Majesty’s Stationery Office, London, 1961.

[24] D. Ciampi de Andrade, C. L. Rodrigues, R. Abraham, L. H. Castro, J. A. Livramento, L. R. Machado, et al., "Cognitive Impairment and Dementia in Neurocysticercosis: A Cross-Sectional Controlled Study,” Neurology, Vol. 74, No. 16, 2010, pp. 1288-1295. doi:10.1212/WNL.0b013e3181d9eda6

[25] A. Rosselli, M. Rosselli, A. Ardila and B. Penagos, "Severe Dementia Associated with Neurocysticercosis," International Journal of Neuroscience, Vol. 41, No. 1-2, 1988, pp. 87-95.

[26] M. Diagana, M. E. Cruz, A. Tabo, I. Cruz, M. Dumas and P. M. Preux, "Cognitive Disorders in an Andean Community Located in a Cysticercosis Endemic Zone of Ecuador," Medecine Tropicale, Vol. 65, No. 4, 2005, pp. 343-345.

[27] S. Jha and M. K. Ansari, "Dementia as the Presenting Manifestation of Neurocysticercosis: A Report of Two Patients,” Neurology Asia, Vol. 15, No. 1, 2010, pp. 8387.

[28] A. Biswas, A. Prasad and K. S. Anand, "Cysticercal DeMentia,” Journal of Association of Physicians of India, Vol. 46, No. 6, 1998, p. 569.

[29] R. M. Hoek, R. E. van Kesteren, A. B. Smit, M. de JongBrink and W. P. Geraerts, "Altered Gene Expression in the Host Brain Caused by a Trematode Parasite: Neuropeptide 
Genes Are Preferentially Affected during Parasitosis,” Proceedings of the National Academy of Sciences of the United States of America, Vol. 94, No. 25, 1997, pp. 14072-14076. doi:10.1073/pnas.94.25.14072

[30] A. Vyas, S. K. Kim, N. Giacomini, J. C. Boothroyd and R. M. Sapolsky, "Behavioral Changes Induced by Toxoplasma Infection of Rodents Are Highly Specific to Aversion of Cat Odors," Proceedings of the National Academy of Sciences of the United States of America, Vol. 104, No. 15, 2007, pp. 6442-6447. doi:10.1073/pnas.0608310104
[31] M. M. Bianchin, T. R. Velasco, O. M. Takayanagui and A. C. Sakamoto, "Neurocysticercosis, Mesial Temporal Lobe Epilepsy, and Hippocampal Sclerosis: An Association Largely Ignored," Lancet Neurology, Vol. 5, No. 1, 2006, pp. 20-21. doi:10.1016/S1474-4422(05)70269-6

[32] J. Ramirez-Bermudez, J. Higuera, A. L. Sosa, E. LopezMeza, M. Lopez-Gomez and T. Corona, "Is Dementia Reversible in Patients with Neurocysticercosis?” Journal of Neurology, Neurosurgery \& Psychiatry, Vol. 76, No. 8, 2005, pp.1164-1166. doi:10.1136/jnnp.2004.052126 